\title{
THE LARGE CONDITION FOR RINGS WITH KRULL DIMENSION
}

\author{
ANN K. BOYLE 1
}

\begin{abstract}
A module $M$ with Krull dimension is said to satisfy the large condition if for any essential submodule $L$ of $M$, the Krull dimension of $M / L$ is strictly less than the Krull dimension of $M$. For a right noetherian ring $R$ with Krull dimension $\alpha$ this is equivalent to the condition that every f.g. uniform submodule of $E\left(R_{R}\right)$ with Krull dimension $\alpha$ is critical. It is also shown that if $R$ is right noetherian with Krull dimension $\alpha$ and if $I_{0}$ is a right ideal maximal with respect to $K \operatorname{dim} I_{0}<\alpha$, then $R$ satisfies the large condition if and only if $I_{0}$ is a finite intersection of cocritical right ideals and $I_{0}$ is closed.
\end{abstract}

1. Introduction and definitions. Let $|M|$ denote the Krull dimension of a module $M$. A module $M$ with Krull dimension is said to satisfy the large condition if for any essential submodule $L,|M / L|<|M|$. According to [6,6.1] every semiprime ring with Krull dimension satisfies the large condition. In fact, many properties of semiprime rings with Krull dimension stem from the large condition (see $[1,2.6]$ and $[6, \S 6]$ ) and these properties are, therefore, shared with this class of rings. It is the purpose of this paper to classify rings satisfying the large condition. When possible, results will be stated for modules.

In $\$ 2$ it is shown that if $M$ is a right noetherian module it suffices to consider the finitely generated uniform submodules of $E(M)$ having Krull dimension $|M|$. If these are critical then $M$ satisfies the large condition. This characterizes right noetherian rings which satisfy the large condition.

According to Goldie $[4,1.5]$ if $R$ is a finite dimensional ring, then zero is a finite intersection of maximal complement right ideals. When $R$ is a semiprime ring with Krull dimension these ideals are cocritical. Modules having this property, that zero is a finite intersection of cocritical right ideals, are called semicritical in [1] and it is shown there that semicritical modules satisfy the large condition. Although not every right noetherian module satisfying the large condition is semicritical, as an example in $\$ 3$ shows, this concept is useful in the study of modules satisfying the large condition. Thus $\$ 3$ is devoted to the consideration of semicritical rings and modules. This

Received by the editors August 11, 1977.

AMS (MOS) subject classifications (1970). Primary 16A46; Secondary 16A12, 16A20.

Key words and phrases. The large condition, semicritical module, semiprime ring, nonsingularly $k$-primitive ring.

${ }^{1}$ This research was supported in part by a grant from the University of Wisconsin-Milwaukee. 
yields an internal characterization of right noetherian rings satisfying the large condition.

All rings in this paper are associative with unit and all modules are unital. Unless stated otherwise module will mean right module. The injective hull of a module $M$ is denoted by $E(M)$ and the singular submodule by $Z(M)$. If $M$ is a module and $K$ a submodule such that $M / K$ is critical, then $K$ is said to be cocritical. A certain familiarity with the definitions and basic results concerning Krull dimension as given in [6] is assumed.

2. The large condition. In this section sufficient conditions for a module to satisfy the large condition are obtained. These conditions are shown to characterize right noetherian rings satisfying the large condition.

A module $M_{R}$ is said to be semicritical if there exists a finite set $K_{1}, \ldots, K_{n}$ of cocritical submodules such that $\bigcap_{i=1}^{n} K_{i}=0$.

2.1 Proposition. Let $R$ be a ring with Krull dimension and $M$ be a f.g. $R$-module. If every f.g. uniform submodule $U$ of $E(M)$ with $|U| \leqslant|M|$ is critical, then $M$ is semicritical.

Proof. Let $U_{1} \oplus \cdots \oplus U_{n}$ be an essential direct sum of uniform submodules of $M$. Let $K_{i}$ be a complement of $U_{i}$ containing $U_{1} \oplus \cdots \oplus$ $U_{i-1} \oplus U_{i+1} \oplus \cdots \oplus U_{n}$. Then $K_{i}$ is a maximal complement and consequently $M / K_{i}$ is a f.g. uniform module. Since $U_{i}$ is isomorphic to an essential submodule of $M / K_{i}, E\left(M / K_{i}\right) \cong E\left(U_{i}\right) \subset E(M)$. So by hypothesis $M / K_{i}$ is critical. Clearly $\cap_{i=1}^{n} K_{i}=0$.

A submodule $N$ of a module $M$ is said to be closed in $M$ if $N$ has no proper essential extensions inside $M$.

2.2 Lemma. Let $M$ be a module with $|M|=\alpha$ and $M_{0}$ a closed submodule of $M$ with $\left|M_{0}\right|<\alpha$. If $M / M_{0}$ satisfies the large condition, then $M$ satisfies the large condition.

Proof. Let $L$ be an essential submodule of $M$. According to [5, 1.4], $M_{0}$ closed implies that $L+M_{0} / M_{0}$ is essential in $M / M_{0}$. Therefore $\mid M / L+$ $M_{0}|<\alpha=| M / M_{0} \mid$. Consequently,

$$
\begin{aligned}
|M / L| & =\sup \left\{\left|M / L+M_{0}\right|,\left|L+M_{0} / L\right|\right\} \\
& =\sup \left\{\left|M / L+M_{0}\right|,\left|M_{0} / L \cap M_{0}\right|\right\}<\alpha=|M| .
\end{aligned}
$$

2.3 Lemma. Let $M$ be a finite dimensional module. If $M_{0}$ is a closed submodule of $M$, then $E\left(M / M_{0}\right) \cong E(M) / E\left(M_{0}\right)$.

Proof. Let $K$ be a complement of $M_{0}$. If $M \supset N \supset M_{0}$ and $N \cap K=0$, the finite dimensionality of $M$ implies that $N$ is an essential extension of $M_{0}$. Since $M_{0}$ is closed, $N=M_{0}$. Thus $K \cong K+M_{0} / M_{0}$ is essential in $M / M_{0}$ and $E(K) \cong E\left(M / M_{0}\right)$. Since $E(M) \cong E(K) \oplus E\left(M_{0}\right)$, it follows that $E(K) \cong E\left(M / M_{0}\right) \cong E(M) / E\left(M_{0}\right)$. 
2.4 THEOREM. Let $R$ be a ring with Krull dimension and let $M$ be a right noetherian $R$-module with $|M|=\alpha$. If every f.g. uniform submodule $U$ of $E(M)$ with $|U|=\alpha$ is critical, then $M$ satisfies the large condition.

Proof. Let $M_{0}$ be maximal in the collection of submodules of $M$ with $\left|M_{0}\right|<\alpha$. Clearly $M_{0}$ is unique and contains every submodule of Krull dimension $<\alpha$. Furthermore, every nonzero submodule of $M / M_{0}$ has Krull dimension $\alpha$.

Let $U_{1} \oplus \cdots \oplus U_{n}$ be an essential direct sum of uniform submodules of $M_{0}$. Then $E\left(M_{0}\right) \cong \sum_{i=1}^{n} \bigoplus E\left(U_{i}\right)$. For ease of notation identify these injective hulls. Let $x \in E\left(U_{i}\right) \subset E\left(M_{0}\right)$. Then $x R$ is a f.g. uniform submodule of $E(M)$. If $|x R|=\alpha$, then $x R$ is critical. However, $x R \cap M_{0} \neq 0$ and $\mid x R \cap$ $M_{0} \mid<\alpha$ which is impossible. Necessarily then $|x R|<\alpha$. So if $h \in E\left(M_{0}\right)=$ $\sum_{i=1}^{n} \bigoplus E\left(U_{i}\right)$, say $h=\sum_{i=1}^{n} x_{i}$ with $x_{i} \in E\left(U_{i}\right)$, then $\left|x_{i} R\right|<\alpha$ for all $i$ and this implies $|h R|<\alpha$. Thus every f.g. submodule of $E\left(M_{0}\right)$ has Krull dimension $<\alpha$.

It follows from this that $M_{0}$ is closed. For if $K$ is an essential extension of $M_{0}$ contained in $M$, then $E(K)=E\left(M_{0}\right)$. If $k \in K$, then the above shows that $|k R|<\alpha$. Since $M_{0}$ is maximal among submodules of $M$ having Krull dimension $<\alpha, k R \subset M_{0}$ and consequently $K=M_{0}$. Thus $M_{0}$ is closed in $M$.

Let $E(M)=H \oplus E\left(M_{0}\right)$. If $U$ is a f.g. uniform submodule of $E\left(M / M_{0}\right)$ with $|U| \leqslant\left|M / M_{0}\right|$, then $U$ has Krull dimension $\alpha$. By $2.3 E\left(M / M_{0}\right) \cong$ $E(M) / E\left(M_{0}\right) \cong H$ and thus $U$ is isomorphic to a f.g. uniform submodule of $H \subset E(M)$. By hypothesis, then $U$ is critical. It follows from 2.1 that $M / M_{0}$ is semicritical and by $[1,2.4]$ then $M / M_{0}$ satisfies the large condition. Finally according to 2.2, $M$ satisfies the large condition.

The converse of 2.3 is not true in general. Let $F$ be a field and $R=\left(\begin{array}{ll}F & F \\ 0 & F\end{array}\right)$. Then $U=\left(\begin{array}{cc}F & F \\ 0 & 0\end{array}\right)$ is a uniform $R$-module which contains the simple submodule $S=\left(\begin{array}{ll}0 & F \\ 0 & 0\end{array}\right)$. Clearly $S$ satisfies the large condition. However, $E(S)$ contains the f.g. uniform submodule $U$ having $|U|=|S|=0$ and $U$ is clearly not critical. Thus it is possible to have a right noetherian module $M$ which satisfies the large condition but which has f.g. uniform submodules of Krull dimension $|M|$ in its injective hull which are not critical. The converse to 2.3 , however, is true for rings.

2.5 Corollary. Let $R$ be a right noetherian ring with $|R|=\alpha$. Then $R$ satisfies the large condition if and only if every f.g. uniform submodule $U$ of $E(R)$ with $|U|=\alpha$ is critical.

Proof. Suppose $R$ satisfies the large condition. Let $U=\sum_{i=1}^{n} x_{i} R$ be a f.g. uniform submodule of $E(R)$ with $|U|=\alpha$ and let $0 \neq U_{0} \subset U$. Then $U_{0}$ is essential in $U$ and as a result $I_{i}=\left\{r \in R \mid x_{i} r \in U_{0}\right\}$ is essential in $R$. In as much as $x_{i} R+U_{0} / U_{0} \cong R / I_{i}$, the large condition implies that $\mid x_{i} R+$ 
$U_{0} / U_{0}|<| R \mid=\alpha$. Thus $\left|U / U_{0}\right| \leqslant \sup \left\{\left|x_{i} R+U_{0} / U_{0}\right|: 1 \leqslant i \leqslant n\right\}<\alpha=$ $|U|$. So $U$ is critical.

The converse is a consequence of 2.4 .

2.6 COROLLARY. If $R$ is a right noetherian ring satisfying the large condition then

(1) every f.g. submodule of $E(R)$ with Krull dimension $|R|$ satisfies the large condition;

(2) every f.g. nonsingular $R$-module with Krull dimension $|R|$ satisfies the large condition.

3. Semicritical modules. If $M$ is a semicritical module, then from [1] $M$ satisfies the large condition. However, not every module which satisfies the large condition is semicritical, as can be seen from the following example. Let $F$ be a field, $R_{1}=F[x]$ and $R_{2}=\left(\begin{array}{c}F \\ 0\end{array} \begin{array}{c}F \\ F\end{array}\right)$. Then $R=R_{1} \times R_{2}$ is a right noetherian ring which satisfies the large condition. Since $\left(\begin{array}{ll}F & F \\ 0 & 0\end{array}\right)$ is uniform but not critical over $R$, according to [1,2.3], $R$ is not semicritical. The aim of this section is to distinguish between semicritical rings and those which satisfy the large condition.

A ring $R$ is called $k$-primitive if $R$ has a finitely generated faithful critical module. Rings which are $k$-primitive of Krull dimension $k$ are considered in [1]. It is shown there that these rings are semicritical and, furthermore, that every semicritical ring is a subdirect product of $k$-primitive rings. This relationship between semicritical rings and $k$-primitive rings can be used to characterize right noetherian, nonsingular semicritical rings.

3.1 THEOREM. Let $R$ be a nonsingular right noetherian ring. Then $R$ is semicritical if and only if every finitely generated uniform submodule of $E(R)$ is critical.

Proof. Suppose $R$ is semicritical. By $[1,3.6] R$ is a subdirect product of $k_{i}$-primitive rings of Krull dimension $k_{i}$, say $R / D_{1}, \ldots, R / D_{m}$. Further these $k_{i}$-primitive rings have the property that if $C_{i}$ is a critical right ideal of $R / D_{i}$, then $E\left(C_{i}\right) \cong E\left(C_{j}\right)$ for $i \neq j$. Let $C_{i 1} \oplus \cdots \oplus C_{i n(i)}$ be a maximal direct sum of critical right ideals which miss $D_{i}$. Then $C_{i s}$ is isomorphic to a critical right ideal of $R / D_{i}$ for all $s$. Thus by [2,2.3] $E\left(C_{i s}\right) \cong E\left(C_{i t}\right)$ for all $1 \leqslant s$, $t \leqslant n(i)$, but $E\left(C_{i k}\right) \npreceq E\left(C_{j t}\right)$ for $i \neq j$. Consider $C_{j t} \cap D_{i}$ for $j \neq i$. If $C_{j t} \cap D_{i}=0$, then $C_{j t} \cap \sum_{k=1}^{n(i)} C_{i k} \neq 0$ which implies that $E\left(C_{j t}\right) \cong E\left(C_{i k}\right)$ and this is impossible. Thus $C_{j t} \cap D_{i} \neq 0$. Since $Z\left(R / D_{i}\right)_{R}=0$, necessarily $C_{j t} \subset D_{i}$ for all $j \neq i$ and $1 \leqslant t \leqslant n(j)$.

By [7, 4.9], $E(R) \cong \sum_{i=1}^{m} E\left(C_{i 1}\right) \oplus \cdots \oplus E\left(C_{i n(i)}\right)$. The singular submodule of $R$ is zero and so $Q=E(R)$ is a semisimple artinian ring. As such $Q$ is a direct product of simple artinian rings which are the homogeneous components $E\left(C_{i 1}\right) \oplus \cdots \oplus E\left(C_{i n(i)}\right)$. Thus there exists central idempotents $e_{1}, \ldots, e_{m}$ with $Q=E(R)=\prod_{i=1}^{m} e_{i} Q$ and $e_{i} Q \cong E\left(C_{i 1}\right) \oplus \cdots \oplus E\left(C_{i n(i)}\right)$.

Consider the mapping $\phi_{i}: R \rightarrow e_{i} R$ which is left multiplication by $e_{i}$. Clearly 
$C_{j k} \subset \operatorname{Ker} \phi_{i}$ for all $j \neq i, 1 \leqslant k \leqslant n(j)$. Since $\sum_{j=1 ; j \neq i}^{m} \sum_{k=1}^{n(j)} C_{j k}$ is large in $D_{i}$, $Z\left(e_{i} R\right)_{R}=0$ implies $D_{i} \subset \operatorname{Ker} \phi_{i}$. Finally since $\operatorname{Ker} \phi_{i} \cap C_{i k}=0,1 \leqslant k \leqslant$ $n(i), D_{i}$ is essential in $\operatorname{Ker} \phi_{i}$. However $Z\left(R / D_{i}\right)_{R}=0$ and so by $[5,2.4] D_{i}$ is closed which implies $D_{i}=\operatorname{Ker} \phi_{i}$. Thus $e_{i} R \cong R / D_{i}$ and $e_{i} R$ is therefore a $k_{i}$-primitive ring of Krull dimension $k_{i}$.

Let $U$ be a f.g. uniform $R$-submodule of $Q$. Then $U Q$ is a uniform $Q$-submodule of $Q$ and as a result $U \subset U Q \subset e_{i} Q$ for some $i$. This implies that $e_{i}$ acts as an identity on $U$ and consequently $U$ is a f.g. uniform $e_{i} R$-module contained in $E\left(e_{i} R\right)=e_{i} Q$. By $[2,2.4]$ every finitely generated $e_{i} R$-submodule of $e_{i} Q$ has Krull dimension $\left|e_{i} R\right|$ and by [1,2.7], $U_{e_{i} R}$ is critical. Since the module structure of $U$ as an $R$-module coincides with the module structure of $U$ as an $e_{i} R$-module, $U$ is critical as an $R$-module.

The converse follows from 2.1 .

3.2 COROLlary. If $R$ is a right noetherian semicritical ring with $Z(R)=0$, then every f.g. nonsingular $R$-module is semicritical.

It is now clear when the class of right noetherian semicritical rings coincides with the class of right noetherian rings satisfying the large condition-when every right ideal of $R$ has the same Krull dimension as $R$. A ring $R$ with Krull dimension is called smooth if for every nonzero right ideal $I$, $|I|=|R|$.

3.3 Proposition. Let $R$ be a smooth right noetherian ring. Then $R$ satisfies the large condition if and only if $R$ is semicritical.

Proof. Note that if $R$ is a smooth ring satisfying the large condition then $Z(R)=0$. The remainder follows from 2.4 and 3.1 .

Using this relation between semicritical rings and rings satisfying the large condition, it is now possible to obtain an internal characterization of right noetherian rings satisfying the large condition.

3.4 Proposition. Let $R$ be a right noetherian ring with $|R|=\alpha$ and let $I_{0}$ be a maximal right ideal subject to $\left|I_{0}\right|<\alpha$. Then $R$ satisfies the large condition if and only if $R / I_{0}$ is semicritical and $I_{0}$ is closed.

Proof. It is easy to see that $I_{0}$ is a two-sided ideal and that $R / I_{0}$ is smooth. If $L \supset I_{0}$ is such that $L / I_{0}$ is large in $R / I_{0}$, then $L$ is large in $R$ and so $\left|R / I_{0} / L / I_{0}\right|=|R / L|<|R|=\left|R / I_{0}\right|$. Thus $R / I_{0}$ satisfies the large condition and by 3.3 , then $R / I_{0}$ is semicritical. Since $R / I_{0}$ is smooth and $\left|R / I_{0}\right|=|R|, Z\left(R / I_{0}\right)_{R}=0$ and so by [5, 2.4], $I_{0}$ is closed.

Conversely, if $R / I_{0}$ is semicritical, then by $[1,2.4] R / I_{0}$ satisfies the large condition. Since $I_{0}$ is closed, $R$ satisfies the large condition by 2.2 .

In 3.4 the condition that $I_{0}$ be closed is not redundant. Example 2.2 in [3] is a uniform noetherian ring $R$ having Krull dimension 1 . It contains a simple two-sided ideal which is maximal among right ideals with Krull dimension $<$ 
A. K. BOYLE

1 and this simply is $I_{0}$. Clearly $I_{0}$ is not closed. $R / I_{0}$ is critical and, hence, is semicritical. However, $R$ does not satisfy the large condition since $I_{0}$ is large in $R$ but $\left|R / I_{0}\right|=|R|=1$.

ACKNOWLedgment. The author would like to thank E. H. Feller for a number of very helpful conversations related to this material.

\section{REFERENCES}

1. A. K. Boyle and E. H. Feller, Semicritical modules and $k$-primitive rings, Module Theory, Selected Papers and Problems, Seattle 1977, Lecture Notes in Math., Springer-Verlag, Berlin and New York (to appear).

2. A. K. Boyle, M. G. Deshpande and E. H. Feller, On nonsingularly k-primitive rings, Pacific J. Math. 68 (1977), 303-311.

3. M. G. Deshpande, Structure of right subdirectly irreducible rings. I, J. Algebra 17 (1971), 317-325.

4. A. W. Goldie, Semi-prime rings with maximum condition, Proc. London Math. Soc. 10 (1960), 201-220.

5. K. R. Goodearl, Ring theory, Dekker, New York, 1976.

6. R. Gordon and J. C. Robson, Krull dimension, Mem. Amer. Math. Soc. No. 133 (1973).

7. D. W. Sharpe and P. Vamos, Injective modules, Cambridge Univ. Press, Cambridge, 1972.

Department of Mathematics, University of Wisconsin-Mnlwaukee, Millwaukee, WISCONSIN 53201 